\title{
Verzeichniss der Holzschnitte.
}

Fig. 1. Bindegewebe mit Capillaren. Schematische Zeichnung . . . . . . . 63

Fig. 2. Schnitt. Capillaren-Verschluss durch Blutgerinnsel. Collaterale Ausdehnung. Schematische Zeichnung . . . . . . . . . . .

Fig. 3. Vereinigung der Wundtlichen durch die zellige Neubildung. Plastisch infiltrirtes Gewebe. Schematische Zeichnung. ........ 66

Fig. 4. Reihenfolge der Gefässbildungen; nach A rnold........ 68

Fig. 5. Wunde nit Substanzverlust. Gefässdilatation. Schematische Zeichnung 73

Fig. 6. Granulirende Wunde. Schematische Zeichnung. . . . . . . . . 74

Fig. 7. Fettige Degeneration von Zellen aus Granulationen. Körnchenzellen . 75

Fig. 8. Eiterzellen aus frischem liter. . . . . . . . . . . . . 91

Fig. 9. Micrococcus, Coccoglia, Sticpturoceus, Bacterien, Vibrio, Streptobacteria 96

Fig. 10. Pilzfigur von der Kanilichencornea; nach v. Frisch........ 100

Fig. 11. IIornhautschnitt, 3 Tage nath der Verletang . . . . . . . . . . . 116

Fig. 12. Schnittwunde in der Wange eines Hundes, 24 Stunden nach der Verwundung . . . . . . . . . . . . . 117

Fig. 13. Narbe 9 Tage nach eimem per primam intentionem geheilten Schnitte durch die Lippe eines Kaninchens. . . . . . . . . . . . 118

Fig. 14. Granulationsgewebe. . . . . . . . . . . . . . 118

Fig. 15. Junges Narbengewebe . . . . . . . . . . . . . . . . 119

Fig. 16. Frontalschnitt durch eine Hundezunge; Gefässverhältnisse 48 Stunden nach der Verletzung; nach w y w od zo f f. ........ 120

Fig. 17. Gleicher Schnitt; Gefässbildung 10 Tage nach der Verletzung; nach $w_{\mathrm{y}}$ -

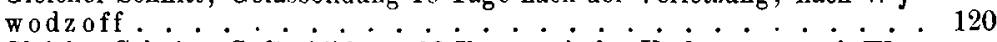

Fig. 18. Gleicher Schnitt; Gefässbildung i6 Tage nach der Verletzung; nach $W_{y}$ -

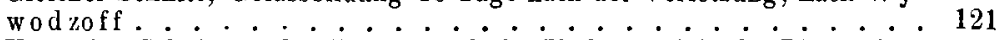

Fig. 19. Vernarbte Schnittwunde (7 Tage nach der Verletzung) in der Lippe eines Hundes. Heilung per primam. Injection der Lymphgefässe . . . . 121

Fig. 20. Muskelfaserenden und Muskelneubildung 8 Tage nach der Verletzung; nach Weber ................... 161

Fig. 21. Regenerationsvorgänge quergestreifter Muskelfasern nach Verletzungen; nach Gussenbauer. . . . . . . . . . . . . 162

Fig. 22. Kaninchennerv 17 und 50 Tage, Froschnerv 30 Tage nach der Durchschneidung; nach Eichborst............. 164

Fig. 23. Kolbige Nervenendigungen an einem älteren Amputationsstumpfe des Oberarms. Amputations-Neurome . . . . . . . . 165

Fig. 24. In der Continuität unterbundene Arterie. Thrombus: nach Froriep 167

Fig. 25. Frischer Thrombus im Querschnitte ............ 168

Fig. 26. Sechstägiger Thrombus im Querschnitte. . . . . . . . . . 168

Fig. 27. Zehntägiger Thrombus . . . . . . . . . . . . 169 
Fig. 28. Vollständig organisirter Thrombus in der Art. tibialis postica des Menschen

Fig. 29. Lüngsschnitt des unterbundenen Endes der Art. crualis eines Hundes; nach 0 . Weber.

Fig. 30. Stück eines Querschnittes der V. femoralis vom Menschen mit organisirtem, vascularisirtem Thrombus. . . . . . . . . . .

Fig. 31. A. carotis eines Kaninchens, 6 Wochen nach der Unterbindung injicirt;

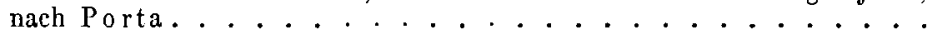

Fig. 32. A. carotis einer Ziege, 35 Monate nach der Unterbindung injicirt; nach

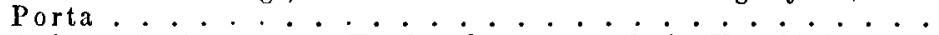

Fig. 33. A. femoralis eines grossen Hundes, 3 Monate nach der Unterbindung injicirt; nach Porta. . . . . . . . . . . . . .

Fig. 34. Seitlich verletzte Arterie mit Gerinnsel, 4 Tage nach der Verwundung; nach Porta. . . . . . . . . . . . . 182

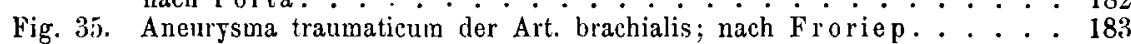

Fig. 36. Varix aneurysmaticus; nach Bell ............ 184

Fig. 37. Aneurysina varicosum: nach Dorsey. . . . . . . . . . . . 184

Fig. 38. Körniges und krystallinisches Hämatoidin. . . . . . . . . . . . . . 197

Fig. 39. Abstossungsprocess abgestorbenen Bindegewebes bei Quetschwunden. . 210

Fig. 40. Ausgerissener Mittelfinger mit sämintlichen Sehnen. . . . . . . . . . 228

Fig. 41. Centrales Ende einer durchrissenen Art. brachialis . . . . . . . . 228

Fig. 42. Ausgerissener Arm mit Scapula und Clavicula. . . . . . . . . . . 228

Fig. 43. 3 Tage alte Fractur eines Kaninchenknochens ohne Iislocation . . . 246

Fig. 44. 10 Tage alte Fractur der Tibia eines Hundes. Nach Stanley. . . 246

Fig. 45. Geheilte Fractur der Fibula. . . . . . . . . . . . . 250

Fig. 46. Fractur der Femurdiaphyse, mit winkeliger Verschiebung geheilt . . . 250

Fig. 47. Dislocirte Fractur des Femur mit reichlichem äusseren Callus. . . . 250

Fig. 48. Schematische Nachbildung eines Sagittalschnittes einer mit winkeliger Uebereinanderschiebung der Fragmente geheilten Fractur der Diaphyse des Femur. Nach J. Wo lff . . . . . . . . . . . . 251

Fig. 49. Querschnitt der Humerusfractur eines Kindes. Nach Thierfelder.

Fig, 50. Schnitt durch einen 52 Stunden alten Callus nach Fractur der Ulna vom Kaninchen. Beginnende Bildung von osteoidem Gewebe. - $\cdot \dot{*} \cdot$.

Fig. 51. Künstlich injicirter äusserer Callus von geringer Dicke an der Oberfläche
ciner Kaniuchen-Tibia in der Nähe einer 5 Tage alten Fractur. .

Fig. 52. Querschnitt durch einen 40 Tage alten Callus nach Fractur der Tibia vom Kaninchen. Resorbtion an der Markhöhle durch Vermittlung von Riesenzellen, Apposition von Knochengewebe am Perioste. Nach

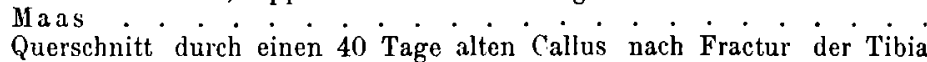

Fig. 53. Querschnitt durch einen 40 Tage alten (allus nach Fractur der Tibia
vom Kaninchen. Resorption des ausseren (Periost-) Callus durch Bil-
dung von Markrumen mit Riesenzellen. Nach Ma as . . .

Fig. 53. Querschnitt durch einen 40 Tage alten (allus nach Fractur der Tibia
vom Kaninchen. Resorption des ausseren (Periost-) Callus durch Bil-
dung von Markrumen mit Riesenzellen. Nach Ma as . . .

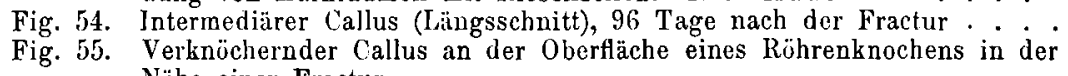
Nähe einer Fractur. . . . . . . . . . . . 259

Fig. 56. Splitterfracturen durch Chassepot- und Zündnadelgewehr-Projectile . . 349

Fig. 57. Blitafiguren; nach Stricker . . . . . . . . . . 360

Fig. 58. Epithelialschicht auf einer catarrhalisch afficirten Conjunetiva; nach Rind fleisch .................. 384

Fig. 59. Entzündlich infiltrirtes Bindegewebe, Einschmelzung der Fasern. . . 388

Fig. 60. A bscessbildung . . . . . . . . . . . . . . . . 390

Fig. 61. Eitrige Infiltration des Panniculus adiposus . . . . . . . . . . . . 391

Fig. 62. Blutgefässe einer Abscesswand . . . . . . . . . . . . . . . . . . 392

Fig. 63. Venenthrombose . . . . . . . . . . . . . . . 469

Fig. 64. Wundfiebercurve. . . . . . . . . . . . . . 475

Fig. 65. Wundfiebercurve nach einer Handgelenkresection.. .476

Fig. 66. Rothe und weisse Blutkörperchen aus der Zwerchfellvene einer sephthämischen Maus. - Bacillen des malignen Oedems vom Kaninchen. Nach R. Koch . . . . . . . . . . . . . .

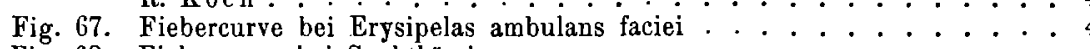

Fig. 68. Fiebercurve bei Sephthämie .
Fig. 69. Gefäss aus der Rindensubstanz der Niere von einem pyohämischen Ka-

Fig. 70. Rothe Blutkörperchen und Bacillen im Milzbrandblut einer Maus. Milz- 
brand, Kaninchen. Gefïss einer Darmzotte mit massenhaften Bacillen;

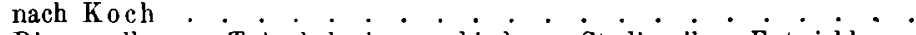

Fig. 71. Riesenzellen aus Tuberlieln in verschiedenen Stadien ihrer Entwicklung; nach Langhans Kleinste Tuberkel iu Setz, kleinste Tuberkel an einer Hirnarterie; nach

Fig. 72. Kleinste Tuberkel itu Netz, kleinste Tuberkel an einer Hirnarterie; nach Kundrat . . . . . . . . . . . . . . . . 555

Fig. 73. Kleinster Tuberkel einer Hinarterie; uach Rindfleisch. . . . . . 557

Fig. 74. Tuberkelbacillen, nach $K \circ \mathrm{ch} . . . . . . . . . . . . . . . . .562$

Fig. 75. Actinomykose, nach Ponfick . . . . . . . . . . . . . . . . . . 580

Fig. 76. Blutgefässnetz üppiger Granulationsknöpfchen; nach Thiersch . . . 598

Fig. 77. Caries superficialis der Tibia; nach Follin........... . 614

Fig. 78. Durchschnitt eines cariösen Knochentheils . . . . . . . . . . . . 615

Fig. 79. Ostitis malacissans . . . . . . . . . . . . . . . . . . . . 621

Fig. 80. Schwund und Kalksalze aus den peripherischen Theilen der Knochenbalken bei Ostitis malacissans; nach Rindfleisch........ 622

Fig. 81. Sklerosirte Knochen; nach Follin . . . . . . . . . . . . . 623

Fig. 82. Verkäster ostitischer Herd in den Rückenwirbeln eines Mannes . . . 627

Fig. 83. Zerstörung der Wirbel durch multiple tuberculose Periostitis und Ostitis anterior .. . . . . . . . . . . . . . . 629

Fig. 84. Nekrose der Tibia. Schematische Zeichnung. . . . . . . . . . . . 654

Fig. 85. Späteres Stadium von Fig. 84.................... . . 656

Fig. 86. Späteres Stadium von Fig. 85. . . . . . . . . . . . . . . . . . 656

Fig. 87. Totale Nekrose des Fernur. . . . . . . . . . . . . . . . . . 658

Fig. 88. Totale Nekrose der Tibia . . . . . . . . . . . . . . . . . . . . . . 658

Fig. 89. Nekrose der unteren lä̈lfte der Diaphyse des Femur mit Lösung des Epiphysenknorpels and Perforation der Haut. . . . . . . . . 659

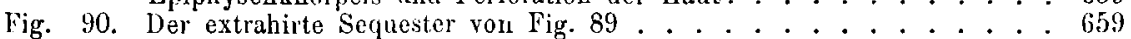

Fig. 91. Partielle Nekrose eines Röhrenknochens. Schematische Zeichnung. . 661

Fig. 92. Späteres Stadium you Fig. 91 . . . . . . . . . . . . . . 661

Fig. 93. Späteres Stadium von Fig. 92 . . . . . . . . . . . . . . . . . . . . . 661

Fig. 94. Regeneration der Scapuli nach Resection. . . . . . . . . . . . (jit2

Fig. 95. Typische Formen von rhachitischen Verkrümmungen der Unterschenkel 673

Fig. 96. Knochenverbiegungen bei Osteomalacie; nach Morand . . . . . . 677

Fig. 97. Schematischer Durchschnitt eines Knicgelenkes mit fungöser Gelenkentzündung . . . . . . . . . . . . . . . . . . 688

Fig. 98. Degeneration des Knorpels bei pannöser Synovitis; nach 0 . Weber 690

Fig. 99. Atonische Knorpelulccrationen aus dem Kniegelenk. . . . . . . . . 691

Fig. 100. Subchondrale granulöse Ostitis am Talus . . . . . . . . . . . . . . . 692

Fig. 101. Degeneration des Knorpels bei Arthritis deformans : nach 0 . Weber. 717

Fig. 102-104. Osteophytenauflagerungen auf Gelenkenden. . . . . . . . . 718

Fig. 105. Schematische Darstellung eines Ganglion . . . . . . . . . . . . . 729

Fig. 106. Synovialhernien am Kniegelenk; nach W. Gruber. . . . . . . . . . 731

Fig. 107. Vielfache Gelenkkürper im Ellenbogengelenk; nach Cruveil hier . . 735

Fig. 108. Bandartige Verwachsungen an einem resecirten Ellenbogengelenke . . 740

Fig. 109. Ankylose durch Knochennarben... . . . . . . . . . . . . 740

Fig. 110 u. 111. Frontalschnitte des Schultergelenkes in verschiedenen Stellungen 741

Fig. 112. Schrumpfung der Fascia lata bei Coxitis : nach Froriep . . . . . 767

Fig. 113 u. 114 . Narbencontracturen nach Verbrennungen. . . . . . . . . . . . . 768

Fig. 115. Subcutan durchschnittene Sehne am vierten'Tage. Schematische Zeichnung 773

Fig. 116. Varices im Gebiet der V. saphena . . . . . . . . . . . . 780

Fig. 117. Aneurysma cirsoideum der Kopfhaut: nach Breschet. . . . . . 788

Fig. 118. Fibrom des Uterus . . . . . . . . . . . . . . . . . . . 831

Fig. 119. Aus einem Myo-Fibjom des Uterus. . . . . . . . . . . . . . . . . . . . . . 832

Fig. 120. Gefässnetze aus Fibromen. . . . . . . . . . . . . . . . . . . . 833

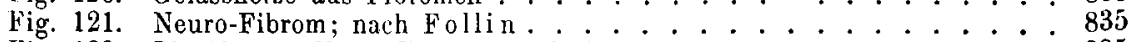

Fig. 122. Plexiformes Neuro-Fibrom; nach P. Bruns . . . . . . . . . . . . . . 835

Fig. 123. Aussergewöhnliche Formen von Knorpelgewebe aus Chondromen. . . 844

Eig. 124. Chondrome der Finger . . . . . . . . . . . . . . . . 846

Fig. 125 u. 126. Odontom. . . . . . . . . . . . . . . . . . . . . 848

Fig. 127-130. Osteome . . . . . . . . . . . . . . . . . . . . . . . . . 850

Fig. 131. Plexiformes Angiom (Teleangiectasic) . . . . . . . . . . . . . . . 858

Fig. 132. Cavernöses Angiom . . . . . . . . . . . . . . . . . 858 
Tig. 133. Seite

Fig. 133. Grantationssarkom . . . . . . . . . . . . . . . 868

Fig. 134. Glio-Sarkom; nach Virchow . . . . . . . . . . . . . 868

Fig. 135. Spindelzellensarkom . . . . . . . . . . . . . . . . . 868

Fig. 136 u. 137. Myxosarkom . . . . . . . . . . . . . . . . . . . . 869

Fig. 138. Riesenzellen aus einem Unterkiefersarkom . . . . . . . . . . . . 870

Fig. 139. Riesenzellensarkom mit Cysten und Verknöcherungsherden . . . . . 871

Fig. 140 u. 141. Alveolares Sarkom . . . . . . . . . . . . . . 872

Fig. 142. Villöses Sarkom der Pia mater. . . . . . . . . . . . . . . 873

Fig. 143. Psammom; nach Virch ow . . . . . . . . . . . . . . 874

Fig. 144. Hirngeschwülste; nach Arnold und Rindfleisch . . . . . . . 875

Fig. 145. Cylindrombildung; nach Sattler. . . . . . . . . . . . 876

Fig. 146. Cylindrom der Orbita . . . . . . . . . . . . . . . . . . 876

Fig. 147 u. 148. Osteosarkom der Ulna. . . . . . . . . . . . . . . . . 883

Fig. 149 u. 150. Osteosarkom des Unterkiefers . . . . . . . . . . . . . . . 883

Fig. 15́1. Osteo-Cystosarkom des Femur; nach Péan . . . . . . . . . 884

Fig. 152 u. 153. Periostsarkom der Tibia. . . . . . . . . . . . . . 885

Fig. 154. Adeno-Sarkom der Mamma . . . . . . . . . . . . . . 887

Fig. 155. Lymphom . . . . . . . . . . . . . . . . . . . . . . 891

Fig. 156. Hautwarze . . . . . . . . . . . . . . . . . . . . . . 900

Fig. 157. Adenomatöser Schleimpolyp des Rectum . . . . . . . . . . . . . . 904

Fig. 158. Kropfgeschwulst. Adenom der Schilddrüse . . . . . . . . . . . 906

Fig. 159. Epithelialkrebs des rothen Lippensaums . . . . . . . . . . . . . . 926

Fig. 160. Flacher Epithelialkrebs der Wange . . . . . . . . . . . . . . . 927

Fig. 161. Elemente eines wuchernden Hautcarcinoms . . . . . . . . . . . 928

Fig. 162. Wuchernder Hautkrebs an der Hand . . . . . . . . . . . . . . . 929

Fig. 163. Gefässe aus einem Carcinom des Penis . . . . . . . . . . . . 929

Fig. 164. Zottenkrebs der Harnblase; nach La m b I. . . . . . . . . . . 934

Fig. 165. Krebs aus dem Innern der Nase . . . . . . . . . . . . . . 939

Fig. 166. Krebs des Rectum . . . . . . . . . . . . . . . . . . . . . 939

Fig. 167. Acinöser Krebs der Mamma . . . . . . . . . . . . . . . . . . . 941

Fig. 168. Aus einem weichen Krebs der Mamma . . . . . . . . . . . . . . 942

Fig. 169. Bindegewebsgerüst aus einem Krebs der Mamma . . . . . . . . . . 942

Fig. 170. Tubulärer Krebs der Mamma . . . . . . . . . . . . . . . . 943

Fig. 171. Schrumpfender Krebs der Mamma. . . . . . . . . . . . . . . . . . . 943

Fig. 172. Gefïssnetz eines ganz jungen Brustdrüsenkrebsknotens . . . . . . . 945

Fig. 173. Gefässnetz in einem Brustdrüsenkrebs. . . . . . . . . . . . . . . 945

Fig. 174. Bindegewebsinfiltration eines Krebsknotens der Mamma . . . . . . . 949

Fig. 175. Infiltration des Fettgewebes in der Peripherie eines Brustkrebses . . . 950 